\title{
High Precision Synchronous Acquisition System for the Current Data in Marine Electromagnetic Transmitter
}

\author{
Shuxiang Wang, Ming Deng, Meng Wang \\ Key Laboratory of Geo-detection, Ministry of Education \\ China University of Geosciences (Beijing), \\ Beijing, China
}

\author{
Xianhu Luo \\ Guangzhou Marine Geological Survey, \\ Guangzhou, China
}

\begin{abstract}
Marine electromagnetic transmitter is one of the key devices for marine electromagnetic exploration, the large current transmitted by the device as well as the data collected by the receiver would undergo process and invert calculation to master the shallow resistivity structure under the seabed. The high precision synchronous acquisition system in this literature is the critical step in this procedure. LPC2368 is adopted in this system as main control unit, and GPS time service is adopted to realize the synchronization with the data from receiver in the acquisition procedure. Temperature compensated crystal oscillator, CPLD time synchronization and frequency division technology as well as time synchronization error query method are adopted in the circuit to ensure the time precision would be up to microsecond, then save the data as well as relevant absolute time to $U$ disk with the $\mathrm{CH} 375$ port convert module in real time manner, with the purpose to ensure the completely storage of the current data of marine electromagnetic transmitter. It is proved in the marine experiment that this system has satisfied the designed requirement.
\end{abstract}

Keywords-marine electromagnetic transmitter; synchronous acquisition; high precision; real time storage

\section{INTRODUCTION}

Marine Controlled Source Electromagnetic exploration technology (MCSEM) has turned into one of the critical methods for marine oil and gas exploration in recent years ${ }^{[1]}$. The research and application of MCSEM has been continuously updated and gradual developed since 1980s in the Western countries. ENI-Agip, Shell, BP, ExxonMobil and Statoil as well as other international petroleum companies participated in the research and application of MCSEM actively ${ }^{21}$. ENI-Agip has developed the 2nd generation of MCSEM technology which could be applied in commercial exploration, and carried out exploration projects in Mediterranean area, Mexico gulf, North Atlantic as well as other area with serious seismic interference ${ }^{[3]}$. However the research on marine electromagnetic method in our country before 1990s is blank, there are no findings in the research of marine electromagnetic exploration devices ${ }^{[4]}$. Thanks to the project "seabed electromagnetic exploration technology for natural gas hydrate" (2006AA09A201) leaded by China University of Geosciences (Beijing) with support from 863 project in our

Foundation Program: Funded by China Geological Survey Project (201100307) and the Fundamental Research Fund for Central Universities (2652015407). country, the research on the marine electromagnetic exploration in natural source in our country has expanded to the artificial source stage, which indicates the formal startup of research on MCSEM technology in our country ${ }^{[5]}$. Marine electromagnetic transmitter is one of the critical components in the marine electromagnetic exploration devices which are based on the above technology. The transmitter would induce high power AC large current to the seabed which would be collected by the seabed electromagnetic collection array (receiver), the receiver would combine the data from these two resources with other parameters, then explore the conductivity structure of the medium in the seabed after certain data processing and invert calculation ${ }^{[6]}$. Therefore the high precision synchronous acquisition system for the current data in marine electromagnetic transmitter in this literature is of great significance to the realization of the entire Marine controlled source electromagnetic exploration technology.

In the application of MCSEM technology, the transmitter would synchronize with the receiver, which generally means that the data from these two devices would have the same start time, the less the tome error is, the higher the synchronization precision would be ${ }^{[7]},{ }^{[8]}$. The sampling rate of the high precision synchronous acquisition system designed in this literature is the same with that of the receiver, with the GPS time service, the absolute time of each data point since the startup is the same with that of the receiver. Considering the operation environment of marine electromagnetic transmitter is under the sea, it is also necessary to solve the issue of data synchronization under the condition that the GPS signal failed. When the data from the transmitter and the receiver failed to be synchronized, the data invert calculation in the later stage would make it quite difficult or even impossible to obtain the desired resistivity structure in the shallow seabed. Therefore the high precision synchronous acquisition system for the current data in marine electromagnetic transmitter realized in this literature provides more guarantee to the validity of data process consequent to the science project "Marine controlled source electromagnetic exploration technology for natural gas hydrate", and found the basis for the upgrade and reform for the pit (borehole) -ground high power electromagnetic transmitter. In addition, high precision synchronous acquisition system is required to be widely used in the synchronous measurement in 
power plant, the gird dispatching operation, power system failure inspection and location as well as other conditions, the application prospect is quite promising.

\section{THE DESIGN OF HIGH PRECISION SYNCHRONOUS ACQUISITION SYSTEM}

The general design framework diagram for high precision synchronous acquisition system for the current data in marine electromagnetic transmitter is as in Fig. 1.

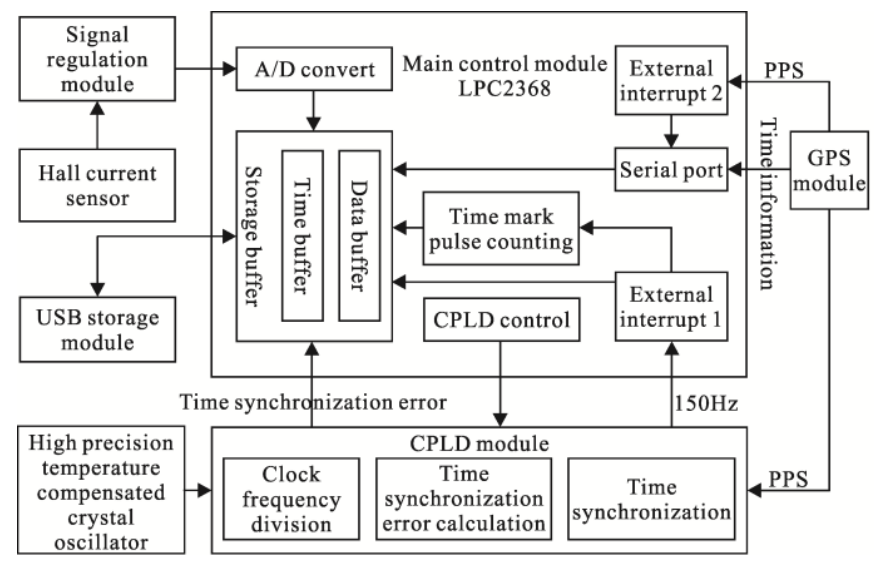

Fig. 1. General design framework diagram for high precision synchronous acquisition system for the current data in marine electromagnetic transmitter

The circuit for hardware in high precision synchronous acquisition system applies modularized design, which is composed of main control module, Complex Programmable Logic Device (CPLD) module, $32.768 \mathrm{MHz}$ high precision temperature compensated crystal oscillator, Hall current sensor, GPS module, USB storage module, and signal regulation module.

As in the general design framework diagram in Fig. 1, the main control module is the core in the system, the current wave shape data collected by Hall current sensor and the corresponding high precision time mark pulse count value would be saved in the U disk via GPS module, CPLD module, USB storage module as well as other external module, for the purpose to form a complete high precision synchronous acquisition system, which in return provides reliable guarantee for the synchronous process for the current wave shape from the marine electromagnetic transmitter and the data collected by the receiver.

Controlled by LPC2368, the CPLD takes pulse per second PPS as reference signal and divides the $32.768 \mathrm{MHz}$ clock signal output from high precision temperature compensated crystal oscillator into $1 \mathrm{~Hz}$ signal and $150 \mathrm{~Hz}$ signal, and then align these two signals with the rising edge of PPS to realize time synchronization. The $150 \mathrm{~Hz}$ signal would be used as time mark signal and input into LPC2368, $1 \mathrm{~Hz}$ would be used in the calculation of error generated from crystal oscillator drift, i.e. time synchronization error value, thus to provide more accurate time mark for the consequent invert calculation.

The current wave shape in electromagnetic transmitter would be collected by Hall current sensor, and then input into A/D converter after processed in regulation module. LPC2368 takes PPS as trigger signal and then create 10 files in TXT format in U disk via USB storage module, then write the time information received by serial port to each TXT files and enable the external interrupt triggered by time mark pulse. Once the $150 \mathrm{~Hz}$ signal triggered the interruption, LPC2368 would read one A/D converted current data, and calculate the number of time mark pulse, then save the two data into buffer. When the buffer is full, LPC2368 will write the data into TXT file.

When the current collection completed, LPC2368 would create end file T.TXT in U disk via USB storage module, then control the CPLD to calculate the time synchronization error value, and write the value with the present time mark pulse number and time information into the T.TXT file.

\section{MARINE EXPERIMENT}

A towed marine electromagnetic transmitter experiment is carried out in the northern area in the South Sea with the "No.6 Marine" from Guangzhou Marine Geological Survey in March 3rd to March 11th, 2015. The startup time for the time synchronization of the data from marine experiment is Greenwich Mean Time (GMT) 2015-03-04-18:40:37:000, the random duration of time is $40000 \mathrm{~ms}$ and data is as in Fig. 2. Transmitter induced artificial source signal (channel 6) and the artificial source signal collected by the receiver (channel 1 5) are showed in Fig. 2. The underwater electromagnetic transmitter induced a mixing frequency supply current the amplitude of which is $200 \mathrm{~A}$, the frequency of which is $0.5 \mathrm{~Hz}$ and $1.5 \mathrm{~Hz}$. We could see clearly from the figure that the transmitting data and received data had been synchronized. 


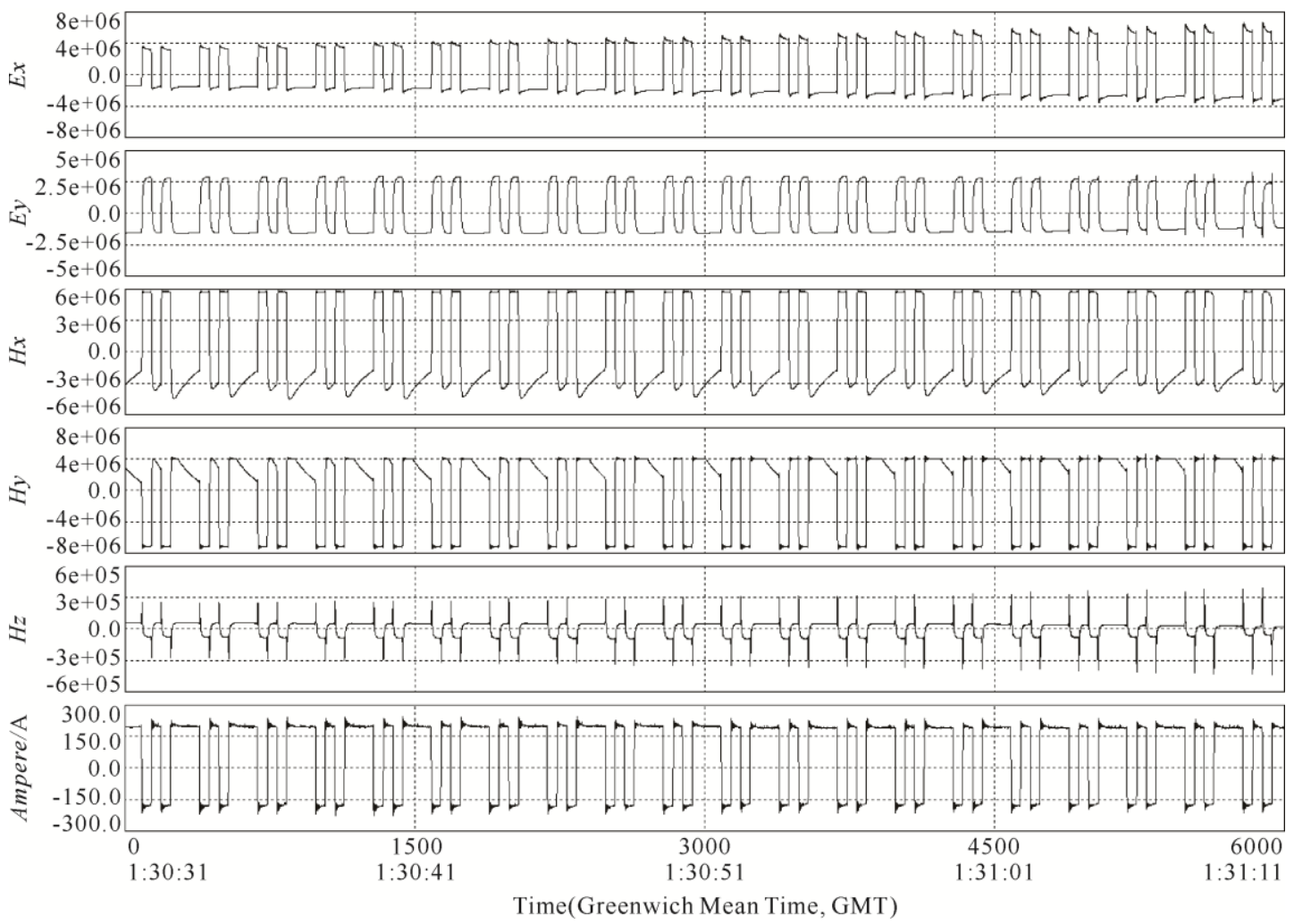

Fig. 2. 23350ms current wave shape data in marine experimen

\section{CONCLUSIONS}

Marine electromagnetic transmitter as a significant component in marine electromagnetic exploration device, the correctness of the data invert calculation is subject to whether the current data and the data in the receiver had been synchronized and the synchronization precision. The design of high precision synchronous acquisition system for the current data in marine electromagnetic transmitter has been realized in this literature, the system takes LPC2368 chip as basis, realizes the sampling with the sampling rate of $150 \mathrm{~Hz}$ with the application of Hall current sensor, regulation module, LPC2368 internal $\mathrm{A} / \mathrm{D}$ and time mark pulse, realizes synchronous collection via GPS module, CPLD time synchronization, error checking, and allow the precision up to $17.82 \mu \mathrm{s} / \mathrm{h}$, adopts $\mathrm{CH} 375$ port chip real time storage of current data and time mark to satisfy the practical operation requirement of marine electromagnetic transmitter thus to provide significant guarantee to the synchronous processing of data from marine electromagnetic transmitter and receiver as well as the further invert calculation.

\section{ACKNOWLEDGMENTS}

I would like to acknowledge the helpful comment on the literature from my tutor Professor Ming Deng and the assistance from the work team.

\section{REFERENCES}

[1] R.N. Edwards, "Marine controlled source electromagnetics: Principles, methodologies, future commercial applications," Surveys in Geophysics, vol. 26, no. 6, pp. 675-700, November 2005.

[2] L. MacGregor, D. Andreis, J. Tomlinson, and N. Barker, "Controlled-source electromagnetic imaging on the Nuggets-1 reservoir," The Leading Edge, vol. 25, no. 8, pp. 984-992, August 2006.

[3] L. MacGregor, and M. Sinha, "Use of marine controlled-source electromagnetic sounding for sub-basalt exploration," Geophysical Prospecting, vol. 48, no. 6, pp. 1091-1106, November 2000.

[4] M. Deng, W.B. Wei, H.D. Tan, S. Jin, H.B. Dong, et al., "Collector for seafloor magnetotelluric data," Chinese Journal of Geophysics, vol. 46, no. 2, pp. 217-223, March 2003.

[5] W.B. Wei, "New advance and prospect of magnetotelluric sounding (MT) in China," Progress in Geophysics, vol. 17, no. 2, pp. 245-254, June 2002.

[6] M. Wang, H.Q. Zhang, Z.L. Wu, Y. Sheng, X.H. Luo, et al., "Marine controlled source electromagnetic launch system for natural gas hydrate resource exploration," Chinese Journal of Geophysics, vol. 56, no. 11, pp. 3708-3717, November 2013.

[7] M. Deng, W.B. Wei, H.D. Tan, S. Jin, and J.W. Deng, "Difficulties in the marine magnetotelluric signal acquisition," Geoscience, vol. 16, no. 1, pp. 94-99, 2002.

[8] M. Wang, M. Deng, Q.S. Zhang, K. Chen, and J.L. Cui, 2009, "The technique of time synchronization operation to control marine electromagnetic emission," Progress in Geophysics, vol. 24, no. 4, pp. 1493-1498, August 2009. 